\title{
Cheeger constants, growth and spectrum of locally tessellating planar graphs
}

\author{
Matthias Keller* \\ Mathematical Institute \\ FSU Jena \\ D-07743 Jena, Germany
}

\author{
Norbert Peyerimhoff ${ }^{\dagger}$ \\ Department of Math. Sciences \\ University of Durham \\ Durham DH1 3LE, UK
}

November 10, 2018

\begin{abstract}
In this article, we study relations between the local geometry of planar graphs (combinatorial curvature) and global geometric invariants, namely the Cheeger constants and the exponential growth. We also discuss spectral applications.
\end{abstract}

\section{Introduction}

A locally tessellating planar graph $G$ is a tiling of the plane with all faces to be polygons with finitely or infinitely many boundary edges. The edges of $G$ are continuous rectifiable curves without self-intersections. Faces with infinitely many boundary edges are called infinigons and occur, e.g., in the case of planar trees. The sets of vertices, edges and faces of $G$ are denoted by $V, E$ and $F$ (see the beginning of Section 2 for precise definitions). The function $d(v, w)$ denotes the combinatorial distance between two vertices $v, w \in V$, where each edge is assumed to have combinatorial length one. For any pair $v, w$ of adjacent vertices we write $v \sim w$.

Useful local concepts of a planar graph $G$ are combinatorial curvature notions. The finest curvature is defined on the corners of $G$. A corner is a pair $(v, f) \in$ $V \times F$, where $v$ is a vertex of the face $f$. The corner curvature $\kappa_{C}$ is defined as

$$
\kappa_{C}(v, f)=\frac{1}{|v|}+\frac{1}{|f|}-\frac{1}{2}
$$

where $|v|$ and $|f|$ denote the degree of the vertex $v$ and the face $f$. If $f$ is an infinigon, we set $|f|=\infty$ and $1 /|f|=0$. The curvature at a vertex $v \in V$ is given by

$$
\kappa(v)=\sum_{f: v \in f} \kappa_{C}(v, f)=1-\frac{|v|}{2}+\sum_{f: v \in f} \frac{1}{|f|} .
$$

*e-mail: m.keller@uni-jena.de

†e-mail: norbert.peyerimhoff@durham.ac.uk 
For a finite set $W \subset V$ we define $\kappa(W)=\sum_{v \in W} \kappa(v)$ and the average vertex curvature by

$$
\bar{\kappa}(W)=\frac{1}{|W|} \kappa(W)
$$

These combinatorial curvature definitions arise naturally from considerations of the Euler characteristic and tessellations of closed surfaces, and they allow to prove a combinatorial Gauß-Bonnet formula (see [BP1, Theorem 1.4]). Similar combinatorial curvature notions have been introduced by many other authors, e.g., St, Gro, Woe, Hi]. Let us already mention two global geometric consequences of the curvature sign:

- In DeVMo it was proved that strictly positive vertex curvature implies finiteness of a graph, thus proving a conjecture of Higuchi (which is a discrete analogue of Bonnet-Myers Theorem in Riemannian geometry). This question was investigated before by Stone [St].

- The cut locus $\operatorname{Cut}(v)$ of a vertex $v$ consists of all vertices $w \in V$, at which $d_{v}:=d(v, \cdot)$ attains a local maximum, i.e., we have $w \in \operatorname{Cut}(v)$ if $d_{v}\left(w^{\prime}\right) \leq d_{v}(w)$ for all $w^{\prime} \sim w$. If $G$ is a plane tessellation with non-positive corner curvature, then $G$ is without cut locus, i.e., we have $\operatorname{Cut}(v)=\emptyset$ for all $v \in V$. This fact can be considered as a combinatorial analogue of the Cartan-Hadamard Theorem (for a proof and more details see BP2, Theorem 1]).

For a finite subset $W \subset V$, let $\operatorname{vol}(W)=\sum_{v \in W}|v|$. We consider the following two types of Cheeger constants:

$$
\alpha(G)=\inf _{\substack{W \subseteq V,|W|<\infty}} \frac{\left|\partial_{E} W\right|}{|W|} \text { and } \widetilde{\alpha}(G)=\inf _{\substack{W \subseteq V,|W|<\infty}} \frac{\left|\partial_{E} W\right|}{\operatorname{vol}(W)},
$$

where $\partial_{E} W$ is the set of all edges $e \in E$ connecting a vertex in $W$ with a vertex in $V \backslash W$. The quantity $\alpha(G)$ is called the physical Cheeger constant and $\widetilde{\alpha}(G)$ the geometric Cheeger constant of the graph $G$. The attributes physical and geometric are motivated by the fact that these constants are closely linked to two types of Laplacians (see, e.g., $\mathrm{Ke}, \mathrm{We}$ ) and that the first type is used in the community of Mathematical Physics whereas the second appears frequently in the context of Spectral Geometry. Cheeger constants are invariants of the global asymptotic geometry. They are important geometric tools for spectral considerations (both in setting of graphs and of Riemannian manifolds) and play a prominent role in the topic of expanders and Ramanujan graphs (see HLW for a very recommendable survey on this topic).

Natural model spaces are the $(p, q)$-regular plane tessellations $G_{p, q}$ : every vertex in $G_{p, q}$ has degree $p$ and every face has degree $q$. (In the case $\frac{1}{p}+\frac{1}{q}<\frac{1}{2}$, $G_{p, q}$ can be realised as a regular tessellation of the Poincaré disc model of the hyperbolic plane by translates of a regular compact polygon.) The graphs $G_{p, q}$ can be considered as discrete counterparts of constant curvature space forms in Riemannian Geometry. The Cheeger constants of these regular graphs are explicitely given: 
Theorem (see [HJL, HiShi, HiShi2]). Let $\frac{1}{p}+\frac{1}{q} \leq \frac{1}{2}$. Then

$$
\widetilde{\alpha}\left(G_{p, q}\right)=\frac{p-2}{p} \sqrt{1-\frac{4}{(p-2)(q-2)}} .
$$

Let us now leave the situation of regular tessellations. It is known that the Cheeger constants of general negatively curved planar graphs are strictly positive (see [Do, [Hi] and Woe]). Moreover, for infinite planar graphs $G$ with $|v| \geq p$ and $|f| \geq q$ for almost all vertices and faces and $c:=\frac{1}{2}-\frac{1}{p}-\frac{1}{q}>0$, the following estimate was shown in $\underline{\mathrm{MO}}$ :

$$
\alpha(G) \geq \frac{2 p q c}{3 q-8} .
$$

Next we introduce a bit of notation before we state our explicit lower Cheeger constant estimates. The variables $p, q$ in this paper always represent a pair of numbers $3 \leq p, q \leq \infty$ satisfying $\frac{1}{p}+\frac{1}{q} \leq \frac{1}{2}$ (note that we use $1 / \infty=0$ ). For such a pair $(p, q)$, let

$$
C_{p, q}:= \begin{cases}1, & \text { if } q=\infty \\ 1+\frac{2}{q-2}, & \text { if } q<\infty \text { and } p=\infty \\ \left(1+\frac{2}{q-2}\right)\left(1+\frac{2}{(p-2)(q-2)-2}\right), & \text { if } p, q<\infty\end{cases}
$$

Then we have

Theorem 1 (Cheeger constant estimate). Let $G=(V, E, F)$ be a locally tessellating planar graph such that $|v| \leq p \forall v \in V$ and $|f| \leq q \forall f \in F$. (Note that $p=\infty$ or $q=\infty$ means no condition on the vertex of face degrees.) Let $C_{p, q}$ be defined as in (3).

(a) Assume that $C:=\inf _{v \in V}-\kappa(v)$ is strictly positive. Then

$$
\alpha(G) \geq 2 C_{p, q} C .
$$

(b) Assume that $c:=\inf _{v \in V}-\frac{1}{|v|} \kappa(v)$ is strictly positive. Then

$$
\widetilde{\alpha}(G) \geq 2 C_{p, q} c .
$$

The above estimates are sharp in the case of regular trees (in which case $q=\infty$ ).

The proof of this theorem is given in Section 2, Observe that the constant $C_{p, q} \geq 1$ in (3) becomes largest if the graph $G$ in Theorem 1 has both finite upper vertex and face degrees. (A shorter expression for $C_{p, q}$ is $\frac{q(p-2)}{(p-2)(q-2)-2}$, which we have to interpret in the right way if $q=\infty$ or $p=\infty$.)

Let us study our estimate in the regular case $G=G_{p, q}$ : In this case our estimate yields

$$
(p-2)\left(1-\frac{2}{(p-2)(q-2)-2}\right) \leq \alpha\left(G_{p, q}\right)
$$


On the other hand, a straightforward calculation leads to the following upper inequality

$$
\alpha\left(G_{p, q}\right)=(p-2) \sqrt{1-\frac{4}{(p-2)(q-2)}} \leq(p-2)\left(1-\frac{2}{(p-2)(q-2)-1}\right),
$$

which shows that our lower bound is very close to the correct value. Mohar's estimate (22) in this situation coincides with ours in the particular case $(p, q)=$ $(\infty, 3)$, and becomes considerably weaker for $q \geq 4$ or $p<\infty$.

Remark. Any infinite connected graph $G=(V, E)$ with $|v| \leq p$ has physical Cheeger constant $\alpha(G) \leq p-2$. To see this, choose an infinite path $v_{0}, v_{1}, v_{2}, \ldots$ and let $W_{n}:=\left\{v_{0}, v_{1}, \ldots, v_{n}\right\}$. Then we have

$$
\frac{\left|\partial_{E} W_{n}\right|}{\left|W_{n}\right|} \leq \frac{2(p-1)+(n-1)(p-2)}{n+1}
$$

which implies

$$
\alpha(G) \leq \lim _{n \rightarrow \infty} \frac{\left|\partial_{E} W_{n}\right|}{\left|W_{n}\right|}=p-2 .
$$

The same arguments show $\alpha\left(T_{p}\right)=p-2$ and $\widetilde{\alpha}\left(T_{p}\right)=\frac{p-2}{2}$, where $T_{p}$ denotes the p-regular infinite tree.

Next, we turn to another global asymptotic invariants related to the growth of an infinite graph $G=(V, E)$. For a fixed center $v_{0} \in V$, let $S_{n}=S_{n}\left(v_{0}\right)=$ $\left\{v \in V \mid d\left(v_{0}, v\right)=n\right\}$ be the spheres of radius $n$ and $\sigma_{n}=\left|S_{n}\right|$. The growth series for $\left(G, v_{0}\right)$ is the formal power series $f_{G, v_{0}}(z)=\sum_{n=0}^{\infty} \sigma_{n} z^{n}$ and the exponential growth $\mu\left(G, v_{0}\right)$ is given by

$$
\mu\left(G, v_{0}\right)=\limsup _{n \rightarrow \infty} \frac{\log \sigma_{n}}{n} .
$$

By Cauchy-Hadamard, the growth series represents a well-defined function in the open complex ball of radius $e^{-\mu\left(G, v_{0}\right)}$. In many cases the exponential growth does not depend on the choice of $v_{0}$. If this is the case, we simply write $\mu(G)$.

Of particular importance in the study of the growth series $f_{G, v_{0}}$ are recursion formulas for the sequence $\sigma_{n}$. In this paper, we consider the case of $q$-face regular plane tessellations $G=(V, E, F)$ (i.e., $|f|=q$ for all $f \in F)$. Before stating our result in terms of average curvatures over spheres $\bar{\kappa}\left(S_{n}\right)=\frac{\kappa\left(S_{n}\right)}{\sigma_{n}}$ we need, again, some notation: For $3 \leq q<\infty$ let $N=\frac{q-2}{2}$ if $q$ is even and $N=q-2$ if $q$ is odd, and

$$
b_{l}= \begin{cases}\frac{4}{q-2} & \text { if } q \text { is even, } \\ \frac{4}{q-2} & \text { if } q \text { is odd and } l \neq \frac{N-1}{2} \\ \frac{4}{q-2}-2 & \text { if } q \text { is odd and } l=\frac{N-1}{2}\end{cases}
$$

for $0 \leq l \leq N-1$.

Theorem 2 (Growth recursion formulas). Let $G=(V, E, F)$ be a q-face regular plane tessellation without cut locus, $S_{n}=S_{n}\left(v_{0}\right)$ for some $v_{0} \in V$ and $\sigma_{n}=\left|S_{n}\right|$. 
Let $N$ and $b_{l}$ be defined as above (see (4)). Moreover, let $\kappa_{n}=\frac{2 q}{q-2} \bar{\kappa}\left(S_{n}\right)$. Then we have the following $(N+1)$-step recursion formulas for $n \geq 1$ :

$$
\sigma_{n+1}= \begin{cases}\sigma_{1}+\sum_{l=0}^{n-1}\left(b_{l}-\kappa_{n-l}\right) \sigma_{n-l} & \text { if } n<N, \\ \sum_{l=0}^{N-1}\left(b_{l}-\kappa_{N-l}\right) \sigma_{N-l} & \text { if } n=N, \\ -\sigma_{n-N}+\sum_{l=0}^{N-1}\left(b_{l}-\kappa_{n-l}\right) \sigma_{n-l} & \text { if } n>N .\end{cases}
$$

A proof of this theorem is given in Section 3 . Note that the constants $\kappa_{k}$ are zero for the regular flat tessellations $G_{3,6}, G_{4,4}$ and $G_{6,3}$. The constants $\kappa_{k}$ in (5) can, therefore, be considered as curvature correction terms for general non-flat tessellations.

In the special case of $(p, q)$-regular graphs $G=G_{p, q}$, the terms $b_{l}-\kappa_{k}$ all coincide with the constant $p-2$ except in the case if $q$ is odd and $l=\frac{N-1}{2}$, when we have $b_{l}-\kappa_{k}=p-4$. In this case, Theorem 2 is equivalent to the fact that $h_{p, q} f_{G, v_{0}}=g_{p, q}$ with

$$
\begin{aligned}
h_{p, q} & =1+2 z+\cdots+2 z^{N}+z^{N+1}, \\
g_{p, q} & =1-(p-2) z-\cdots-(p-2) z^{N}+z^{N+1},
\end{aligned}
$$

if $q$ is even, and

$$
\begin{aligned}
& h_{p, q}=1+2 z+\cdots+2 z^{\frac{N-1}{2}}+4 z^{\frac{N+1}{2}}+2 z^{\frac{N+3}{2}}+\cdots+2 z^{N}+z^{N+1}, \\
& g_{p, q}=1-(p-2) z-\cdots-(p-4) z^{\frac{N+1}{2}}-\cdots-(p-2) z^{N}+z^{N+1},
\end{aligned}
$$

if $q$ is odd. This agrees with results of Cannon and Wagreich CaWa and Floyd and Plotnick [FP, §3] that the growth function $f_{G, v_{0}}$ is the rational function $h_{p, q} / g_{p, q}$. Moreover, it was shown in [CaWa] and [BaCS] that the denominator polynomial $g_{p, q}$ for $\frac{1}{p}+\frac{1}{q}<\frac{1}{2}$ is a reciprocal Salem polynomial, i.e., its roots lie on the complex unit circle except for two positive reciprocal real zeros $\frac{1}{x_{p, q}}<$ $1<x_{p, q}<p-1$. This implies that the exponential growth coincides with $\log x_{p, q}$, i.e.,

$$
\mu\left(G_{p, q}\right)=\log x_{p, q}<\log (p-1)=\mu\left(T_{p}\right) .
$$

(An even more precise decription of the growth of the sequence $\sigma_{n}$ is given in [BaCS, Cor. 3].) Of course, it is desirable to know more about the explicit value of $\mu\left(G_{p, q}\right)=\log x_{p, q}$. Since $g_{p, q}$ is divisible by $z^{2}-\left(p-\frac{4}{q-2}\right) z+1$ in the case $q=3,4,6$, we have

Proposition 1.1. Let $q \in\{3,4,6\}$. Then

$$
\mu\left(G_{p, q}\right)=\log \left(\frac{p}{2}-\frac{2}{q-2}+\sqrt{\left(\frac{p}{2}-\frac{2}{q-2}\right)^{2}-1}\right) .
$$

In most of the cases the polynomial $g_{p, q}$ is essentially irreducible (expect for some small well known factors; see [BaCS, Thm. 1]) and there is no hope to have an explicit expression for its largest zero $x_{p, q}>1$. A direct consequence of the isoperimetric inequality in [BP1, Cor. 5.2] is the following lower estimate of $\log x_{p, q}$ : 
Proposition 1.2. Let $G_{p, q}$ be non-positively curved, i.e.,

$$
C=-\kappa(v)=p\left(\frac{1}{p}+\frac{1}{q}-\frac{1}{2}\right) \geq 0 \quad \forall v \in V,
$$

then we have

$$
\mu\left(G_{p, q}\right)=\log x_{p, q} \geq \log \left(1+\frac{2 q}{q-1} C\right) .
$$

Note that (7) implies $\lim _{q \rightarrow \infty} \mu\left(G_{p, q}\right)=\mu\left(T_{p}\right)=\log p-1$ for all $p \geq 3$.

Remark. The Mahler measure $M(g)$ of a monic polynomial $g \in \mathbb{Z}[z]$ with integer coefficients is given by the product $\prod\left|z_{i}\right|$, where $z_{i} \in C$ are the roots of $g$ of modulus $\geq 1$. Lehmer's conjecture states that for every such $g$ with $M(g)>1$ we have

$$
M(g) \geq M\left(1-z+z^{3}-z^{4}+z^{5}-z^{6}+z^{7}-z^{9}+z^{10}\right) \approx 1.1762 \ldots
$$

Thus (77) yields an explicit lower estimate for the Mahler measure of the polynomials $g_{p, q}(z)$.

Let us now return to general $q$-face regular tessellations. We conclude from Theorem 2 .

Theorem 3 (Curvature/Growth comparison). Let $G=(V, E, F)$ and $\widetilde{G}=$ $(\widetilde{V}, \widetilde{E}, \widetilde{F})$ be two q-face regular plane tessellations with non-positive vertex curvature, $S_{n} \subset V$ and $\widetilde{S}_{n} \subset V$ be spheres with respect to the centres $v_{0} \in V$ and $\widetilde{v}_{0} \in \widetilde{V}$, respectively, and $\sigma_{n}=\left|S_{n}\right|$ and $\widetilde{\sigma}_{n}=\left|S_{n}\right|$. Assume that the spherical average curvatures satisfy

$$
\bar{\kappa}\left(\widetilde{S}_{n}\right) \leq \bar{\kappa}\left(S_{n}\right) \leq 0 \quad \forall n \geq 0 .
$$

Then the difference sequence $\widetilde{\sigma}_{n}-\sigma_{n} \geq 0$ is monotone non-decreasing and, in particular, we have $\mu(\widetilde{G}) \geq \mu(G)$.

A proof of this theorem is given in Section 3. (In fact, the proof shows that the vertex curvature conditions in Theorem 3 can be slightly relaxed: It suffices that $G$ has non-positive vertex curvature and that both graphs $G$ and $\widetilde{G}$ are without cut-loci.) This theorem can be considered as a refined discrete counterpart of the Bishop-Günther-Gromov Comparison Theorem for Riemannian manifolds (see, e.g., GaHuLa, Theorem 3.101]). The latter compares volumes of balls in Riemannian manifolds against constant curvature space forms; our discrete counterpart deals with spheres (the result for balls is obtained by adding over spheres) and is more flexible as it allows to use more general comparison spaces.

If we drop the face regularity condition, it is not difficult to derive the following simple tree comparison result. A proof of this result is given in Section 4 .

Theorem 4 (Tree comparison). Let $G=(V, E, F)$ be a locally tessellating planar graph without cut locus satisfying $|v| \leq p$ for all $v \in V$, for some $p \geq 3$. Then

$$
\mu(G) \leq \mu\left(T_{p}\right)=\log (p-1) .
$$


Note that another more involved tree comparison result was obtained by Higuchi Hi2 for (not necessarily planar) infinite vertex-regular graphs with each vertex contained a cycle of uniformly bounded length.

Let us finally discuss some spectral applications (see MW, Woe2 for classical surveys). The (geometric) Laplacian $\widetilde{\Delta}: \ell^{2}(V, m) \longrightarrow \ell^{2}(V, m)$, where $m(v)=|v|$ for $v \in V$ is given by

$$
(\widetilde{\Delta} \varphi)(v)=\frac{1}{|v|} \sum_{w \sim v} \varphi(v)-\varphi(w), \quad\left(\varphi \in \ell^{2}(V, m), v \in V\right) .
$$

The relation between the bottom $\lambda_{0}(G)$ of the spectrum and the bottom $\lambda_{0}^{\text {ess }}(G)$ of the essential spectrum of $\widetilde{\Delta}$ and the Cheeger constant and the exponential growth in the discrete case was presented first by Dodziuk/Kendall DKe and Dodziuk/Karp DKa]. The best estimates are due to K. Fujiwara (see [Fu1] and [Fu2]):

$$
1-\sqrt{1-\widetilde{\alpha}^{2}(G)} \leq \lambda_{0}(G) \leq \lambda_{0}^{\mathrm{ess}}(G) \leq 1-\frac{2 e^{\mu(G) / 2}}{1+e^{\mu(G)}}
$$

which are sharp in the case of regular trees.

An immediate consequence of Theorem [1] and (8) is the following combinatorial analogue of McKean's Theorem (see $\mathrm{McK}$ for the result in the smooth setting):

Corollary 1.3. Let $G=(V, E, F)$ be a locally tessellating planar graph satisfying the vertex and face degree bounds in Theorem 1, Moreover, assume that $c:=\inf _{v \in V}-\frac{1}{|v|} \kappa(v)>0$. Then

$$
1-\sqrt{1-\left(2 C_{p, q} c\right)^{2}} \leq \lambda_{0}(G)
$$

where $C_{p, q}$ is defined in (3). This estimate is sharp in the case of regular trees.

Similarly, Theorem 3, (6) and (8) directly imply

Corollary 1.4. Let $p, q \geq 3$ and $\frac{1}{p}+\frac{1}{q} \leq \frac{1}{2}$. Let $G$ be a $q$-face regular tessellation without cut locus and satisfying $|v| \leq p$ for all vertices. Then

$$
\lambda_{0}^{\mathrm{ess}}(G) \leq 1-\frac{2 \sqrt{x_{p, q}}}{1+x_{p, q}} .
$$

This estimate is sharp in the case of regular trees.

Let us finish this introduction with some general references. It was shown in KLPS that non-positive corner curvature implies non-existence of finitely supported eigenfunctions of all elliptic operator on planar graphs. Lower estimates for the bottom of the essential spectrum in terms of Cheeger constants at infinity or branching rates of general non-planar graphs can be found in [Fu2 and Ura for the geometric Laplacian and in $\mathrm{Ke}$ and Woj, Woj2 for the physical Laplacian $\Delta: D(\Delta) \subseteq \ell^{2}(V) \longrightarrow \ell^{2}(V)$ given by $(\Delta \varphi)(v)=\sum_{w \sim v} \varphi(v)-\varphi(w)$, $\varphi \in D(\Delta), v \in V$. These results show, in particular, absence of the essential spectrum for graphs with curvature converging to infinity outside increasing compact sets, a phenomenon which was first proved in the smooth context of manifolds by DL. 
Acknowledgement: Matthias Keller likes to thank Daniel Lenz who encouraged him to study the connection between curvature and spectral theory. Matthias Keller was supported during this work by sdw. Norbert Peyerimhoff is grateful for the financial support of the TU Chemnitz. Both authors like to thank Ruth Kellerhals and Victor Abrashkin for very useful discussions.

\section{Proof of Theorem 1}

Let us first give precise definitions of some notions used in the introduction. Let $G=(V, E)$ be a planar graph embedded in $\mathbb{R}^{2}$. The faces $f$ of $G$ are the closures of the connected components in $\mathbb{R}^{2} \backslash \bigcup_{e \in E} e$.

We further assume that $G$ has no loops, no multiple edges and no vertices of degree one (leaves). Moreover, we assume that every vertex has finite degree and that every bounded open set in $\mathbb{R}^{2}$ meets only finitely many faces of $G$. We call a planar graph with these properties simple. We call a sequence of edges $e_{1}, \ldots, e_{n}$ a walk of length $n$ if there is a corresponding sequence of vertices $v_{1}, \ldots, v_{n+1}$ such that $e_{i}=v_{i} v_{i+1}$. A walk is called a path if there is no repetition in the corresponding sequence of vertices $v_{1}, \ldots, v_{n}$. A (finite or infinite) path with associated vertex sequence $\ldots v_{i} v_{i+1} v_{i+2} \ldots$ is called a geodesic, if we have $d\left(v_{i}, v_{j}\right)=|i-j|$ for all pairs of vertices in the path. The boundary of a face $f$ is the subgraph $\partial f=(V \cap f, E \cap f)$. We define the degree $|f|$ of a face $f \in F$ to be the length of the shortest closed walk in the subgraph $\partial f$ meeting all its vertices. If there is no such finite walk, we set $|f|=\infty$. Now we present the conditions which have to be satisfied that a planar graph is locally tessellating:

Definition 2.1. A simple planar graph $G$ is called a locally tessellating planar graph if the following conditions are satisfied:

(i) Any edge is contained in precisely two different faces.

(ii) Any two faces are either disjoint or have precisely a vertex or a path of edges in common. In the case that the length of the path is greater than one, then both faces are unbounded.

(iii) Any face is homeomorphic to the closure of an open disc $\mathbb{D} \subset \mathbb{R}^{2}$, to $\mathbb{R}^{2} \backslash \mathbb{D}$ or to the upper half plane $\mathbb{R} \times \mathbb{R}_{+} \subset \mathbb{R}^{2}$ and its boundary is a path.

Note that these properties force the graph $G$ to be connected. Examples are tessellations $\mathbb{R}^{2}$ introduced in $\mathrm{BP} 1, \mathrm{BP} 2$, trees in $\mathbb{R}^{2}$, and particular finite tessellations on the sphere mapped to $\mathbb{R}^{2}$ via stereographic projection.

Now we turn to the proof of Theorem 1] The heart of the proof is Proposition 2.2 below. An earlier version of this proposition in the dual setting is Proposition 2.1 of BP1. We start with a few more preliminary considerations. Let $G=$ $(V, E, F)$ be a locally tessellating planar graph. For a finite set $W \subseteq V$ let $G_{W}=\left(W, E_{W}, F_{W}\right)$ be the subgraph of $G$ induced by $W$, where $E_{W}$ are the edges in $E$ with both end points in $W$ and $F_{W}$ are the faces induced by the graph $\left(W, E_{W}\right)$. Euler's formula states for a finite and connected subgraph $G_{W}$ (observe that $F_{W}$ contains also the unbounded face):

$$
|W|-\left|E_{W}\right|+\left|F_{W}\right|=2 \text {. }
$$


Recall that $\partial_{E} W$ is the set of edges connecting a vertex in $W$ with one in $V \backslash W$. By $\partial_{F} W$, we denote the set of faces in $F$ which contain an edge of $\partial_{E} W$. Moreover, we define the inner degree of a face $f \in \partial_{F} W$ by

$$
|f|_{W}^{i}=|f \cap W| .
$$

We will need two useful formulas which hold for arbitrary finite and connected subgraphs $G_{W}=\left(W, E_{W}, F_{W}\right)$. The first formula is easy to see and reads as

$$
\sum_{v \in W}|v|=2\left|E_{W}\right|+\left|\partial_{E} W\right| .
$$

Since $W$ is finite, the set $F_{W}$ contains at least one face which is not in $F$, namely the unbounded face surrounding $G_{W}$, but there can be more. Define $c(W)$ as the number

$$
c(W)=\left|F_{W}\right|-\left|F_{W} \cap F\right| \geq 1 .
$$

Note that $\left|F_{W} \cap F\right|$ is the number of faces in $F$ which are entirely enclosed by edges of $E_{W}$. Sorting the following sum over vertices according to faces gives the second formula

$$
\begin{aligned}
\sum_{v \in W} \sum_{f \ni v} \frac{1}{|f|} & =\left|F_{W} \cap F\right|+\sum_{f \in \partial_{F} W} \frac{|f|_{W}^{i}}{|f|} \\
& =\left|F_{W}\right|-c(W)+\sum_{f \in \partial_{F} W} \frac{|f|_{W}^{i}}{|f|} .
\end{aligned}
$$

Proposition 2.2. Let $G=(V, E, F)$ be a locally tessellating planar graph and $W \subset V$ be a finite set of vertices such that the induced subgraph $G_{W}$ is connected. Then we have

$$
\kappa(W)=2-c(W)-\frac{\left|\partial_{E} W\right|}{2}+\sum_{f \in \partial_{F} W} \frac{|f|_{W}^{i}}{|f|} .
$$

Proof. By the equations (10), (12) and (9) we conclude

$$
\begin{aligned}
\kappa(W) & =\sum_{v \in W}\left(1-\frac{|v|}{2}+\sum_{f \ni v} \frac{1}{|f|}\right) \\
& =|W|-\left|E_{W}\right|-\frac{\left|\partial_{E} W\right|}{2}+\left|F_{W}\right|-c(W)+\sum_{f \in \partial_{F} W} \frac{|f|_{W}^{i}}{|f|} \\
& =2-c(W)-\frac{\left|\partial_{E} W\right|}{2}+\sum_{f \in \partial_{F} W} \frac{|f|_{W}^{i}}{|f|} .
\end{aligned}
$$

A finite set $W \subset V$ is called a polygon, if $G_{W}$ is connected and if $c(W)=1$. This notion becomes understandable if one looks at the dual setting: Every vertex $v \in W$ corresponds to a face $f^{*}(v) \in F^{*}$ in the dual planar graph $G^{*}=\left(V^{*}, E^{*}, F^{*}\right)$, and $W \subset V$ is a polygon if and only if $\bigcup_{v \in W} \overline{f^{*}(v)} \subset \mathbb{R}^{2}$ is homeomorphic to a closed disc (here $\bar{f}$ denotes the closure of the geometric 
realization of the face $f)$. For $v \in W$, let $|v|_{W}^{e}$ denote the number of edges in $\partial_{E} W$ adjacent to $v$. $|v|_{w}^{e}$ is called the external degree of $v$ (w.r.t. $W$ ). Moreover, let $\partial_{V} W$ be the set of vertices in $W$ with $|v|_{W}^{e} \geq 1$.

Proposition 2.3. Ler $G=(V, E, F)$ be a locally tessellating planar graph satisfying the vertex and face degree bounds in Theorem 1 and $W \subset V$ be a polygon with $|v|_{W}^{e} \leq p-2$ for all $v \in \partial_{V} W$. Then we have

$$
\left|\partial_{E} W\right| \geq 2 C_{p, q}(1-\kappa(W)) .
$$

Moreover, under the assumption of (a) or (b) in Theorem 1, we have

$$
\frac{\left|\partial_{E} W\right|}{|W|} \geq 2 C_{p, q} C \quad \text { or } \quad \frac{\left|\partial_{E} W\right|}{\operatorname{vol}(W)} \geq 2 C_{p, q} \text {, respectively. }
$$

Proof. Observe first that we have the inequality

$$
\sum_{f \in \partial_{F} W}|f|_{W}^{i} \geq\left|\partial_{V} W\right|+\left|\partial_{E} W\right|
$$

This can be seen as follows: Every face $f \in \partial_{F} W$ may have some edges and some isolated vertices in common with the induced graph $G_{W}=\left(W, E_{W}, F_{W}\right)$. Since the vertices of $\partial_{V} W$ are connected in $G_{W}$, there are at least $\left|\partial_{V} W\right|$ pairs $(f, e) \in \partial_{F} W \times E$ with $e \in \partial f \cap E_{W}$. These pairs contribute at least $2\left|\partial_{V} W\right|$ to the left hand sum in (14). At every vertex $v \in \partial_{V} W$, there are $|v|_{W}^{e}-1$ faces of $\partial_{F} W$ which meet $G_{W}$ in the isolated vertex $v$. Adding over all these vertices $v \in \partial_{V} W$, we obtain the total contribution $\left|\partial_{E} W\right|-\left|\partial_{V} W\right|$ to the left hand sum in (14). One easily checks that there is no overlap of both contributions, leading to the above inequality.

Using (14), $\left|\partial_{V} W\right| \geq \frac{1}{p-2}\left|\partial_{E} W\right|,|f| \leq q$ for all $f \in F$, and Proposition 2.2, we obtain

$$
\left|\partial_{E} W\right|\left(\frac{1}{2}-\frac{p-1}{q(p-2)}\right) \geq \frac{\left|\partial_{E} W\right|}{2}-\sum_{f \in \partial_{F} W} \frac{|f|_{W}^{i}}{|f|}=1-\kappa(W),
$$

which yields (13). The second formula of the proposition follows from $-\kappa(W) \geq$ $C|W|$ in case (a) and from $-\kappa(W) \geq c \operatorname{vol}(W)$ in case (b).

Henceforth, let $G=(V, E, F)$ be a locally tessellating planar graph as in Theorem 1. Recall that $C_{p, q}=\frac{q(p-2)}{(p-2)(q-2)-2}$. The conditions $|v| \leq p$ and $|f| \leq q$ for all $v \in V$ and $f \in F$ imply $2 C_{p, q} C \leq p-2$ and $2 C_{p, q} c \leq \frac{p-2}{p}$.

Lemma 2.4. Let $v \in V$ and $W=\{v\}$. Then we have

$$
\frac{\left|\partial_{E} W\right|}{|W|}=|v| \geq 2 C_{p, q} C
$$

and

$$
\frac{\left|\partial_{E} W\right|}{\operatorname{vol}(W)}=1 \geq \frac{p-2}{p}
$$


Proof. The only non trivial inequality is (15). It follows straightforwardly from $\kappa(v) \leq-C$ that

$$
C \leq \frac{q-2}{2 q}|v|-1 \text {. }
$$

This implies that

$$
\begin{aligned}
2 C_{p, q} C & \leq \frac{(p-2)(q-2)}{(p-2)(q-2)-2}|v|-\frac{2 q(p-2)}{(p-2)(q-2)-2} \\
& \leq|v|-\frac{2}{(p-2)(q-2)-2}(q(p-2)-p) .
\end{aligned}
$$

The lemma follows now from the fact that $q(p-2)-p \geq 0$ for $p, q \geq 3$.

Lemma 2.5. Assume that there is a finite set $W \subset V$ such that

$$
\frac{\left|\partial_{E} W\right|}{|W|}<2 C_{p, q} C \leq p-2 \quad \text { or } \quad \frac{\left|\partial_{E} W\right|}{\operatorname{vol}(W)}<2 C_{p, q} c \leq \frac{p-2}{p}, \text { respectively. }
$$

Then there exists a polygon $W^{\prime} \subset V$ with $|v|_{W^{\prime}}^{e} \leq p-2$ for all $v \in \partial_{V} W^{\prime}$, such that

$$
\frac{\left|\partial_{E} W^{\prime}\right|}{\left|W^{\prime}\right|} \leq \frac{\left|\partial_{E} W\right|}{|W|} \quad \text { or } \quad \frac{\left|\partial_{E} W^{\prime}\right|}{\operatorname{vol}\left(W^{\prime}\right)} \leq \frac{\left|\partial_{E} W\right|}{\operatorname{vol}(W)} \text {, respectively. }
$$

Proof. Observe first that we can always find a non-empty subset $W_{0} \subset W$ such that $G_{W_{0}}$ is a connected component of $G_{W}$ and that $\left|\partial_{E} W_{0}\right| /\left|W_{0}\right| \leq\left|\partial_{E} W\right| /|W|$ or $\left|\partial_{E} W_{0}\right| / \operatorname{vol}\left(W_{0}\right) \leq\left|\partial_{E} W\right| / \operatorname{vol}(W)$, respectively. Note that $G_{W_{0}}$ has only one unbounded face. By adding all vertices of $V$ contained in the union of all bounded faces of $G_{w_{0}}$, we obtain a polygon with even smaller isoperimetric constants. Let us denote this non-empty polygon, again, by $W$. By Lemma 2.4 $W$ must have at least two vertices. By connectedness of $G_{W}$ and $|W| \geq 2$, we have $|v|_{W}^{e} \leq p-1$ for all $v \in W$. Assume there is a vertex $v \in \partial_{V} W$ with $|v|_{W}^{e}=p-1$. Let $W^{\prime}:=W \backslash\{v\}$. Then one easily checks that the condition (16) implies

or

$$
\frac{\left|\partial_{E} W^{\prime}\right|}{\left|W^{\prime}\right|}=\frac{\left|\partial_{E} W\right|+2-p}{|W|-1}<\frac{\left|\partial_{E} W\right|}{|W|}
$$

$$
\frac{\left|\partial_{E} W^{\prime}\right|}{\operatorname{vol}\left(W^{\prime}\right)}=\frac{\left|\partial_{E} W\right|+2-p}{\operatorname{vol}(W)-p}<\frac{\left|\partial_{E} W\right|}{\operatorname{vol}(W)}
$$

respectively. Repeating this elimination of vertices with external degree $p-1$, we end up with a polygon $W^{\prime}$ satisfying $|v|_{W^{\prime}}^{e} \leq p-2$ for all $v \in W^{\prime}$ or with $W^{\prime}$ equal to a single vertex. But the latter case is a contradiction to Lemma 2.4

Proof of Theorem 1. Since $2 C_{p, q} C \leq p-2$ or $2 C_{p, q} c \leq \frac{p-2}{p}$, we only have to consider the cases when $\alpha(G)<p-2$ or $\widetilde{\alpha}(G)<\frac{p-2}{p}$, since otherwise there is nothing to prove. Lemma 2.5 states that, if there is a finite $W \subset V$ with

$$
\frac{\left|\partial_{E} W\right|}{|W|}<2 C_{p, q} C \text { or } \frac{\left|\partial_{E} W\right|}{\operatorname{vol}(W)}<2 C_{p, q} c \text {, respectively, }
$$

then there is a polygon $W^{\prime}$ with $|v|_{W^{\prime}}^{e} \leq p-2$ for all $v \in \partial_{V} W^{\prime}$ satisfying the same inequality. But this contradicts to Proposition 2.3 finishing the proof of Theorem 1 . 


\section{Proofs of Theorems 2 and 3}

Let $G=(V, E, F)$ be a $q$-face regular plane tessellation without cut locus, $v_{0} \in V, S_{n}=S_{n}\left(v_{0}\right)$ and $\sigma_{n}=\left|S_{n}\right|$. Recall that we have $N=\frac{q-2}{2}$ if $q$ is even and $N=q-2$ if $q$ is odd. The recursion formulas (5) in Theorem 2 for $n \leq N$ and $n>N$, respectively, require separate proofs. However, both proofs are based on the following results from [BP1, Section 6] (note that these results are presented there in the dual setting of vertex-regular graphs): Proposition 6.3 in BP1 states for $n \geq 1$ that

$$
\kappa\left(B_{n}\right)=1-\frac{q-2}{2 q}\left(\sigma_{n+1}-\sigma_{n}\right)+\sum_{j=2}^{q-2} \frac{q-2 j}{2 q} c_{n}^{j},
$$

where $B_{n}=\left\{v \in V \mid d\left(v_{0}, v\right) \leq n\right\}$ denotes the ball and

$$
c_{n}^{j}=\left|\left\{f \in F|| f \cap\left(V \backslash B_{n}\right) \mid=j\right\}\right|
$$

for $1 \leq j \leq q-1$. Moreover from Lemma 6.2 in BP1] we have the following recurrence relations for $c_{n}^{j}, n \geq 1$, which arise very naturally from the geometric context

(i) $c_{n}^{l}=c_{n-1}^{l+2}$, for $1 \leq l \leq q-3$,

(ii) $c_{n}^{q-2}=c_{n-1}^{2}$,

(iii) $c_{n}^{q-1}=c_{n}^{1}+\sigma_{n+1}-\sigma_{n}=c_{n-1}^{3}+\sigma_{n+1}-\sigma_{n}$.

We first aim at the proof of (5) for $n \leq N$. Let $\tau:\{0,1, \ldots, N\} \rightarrow$ $\{1,2, \ldots, q-1\}$ be defined as

$$
\tau(k)= \begin{cases}q-1-2 k & \text { if } q \text { is even and } 0 \leq k \leq N \\ q-1-2 k & \text { if } q \text { is odd and } 0 \leq k \leq \frac{N-1}{2} \\ 2 q-3-2 k & \text { if } q \text { is odd and } \frac{N+1}{2} \leq k \leq N\end{cases}
$$

Note that $\tau$ is defined precisely in such a way that we have

$$
c_{n+1}^{\tau(k+1)}=c_{n}^{\tau(k)} \quad \text { for } 0 \leq k \leq N-1 \text { and } n \geq 0,
$$

by the recurrence relations (i) and (ii).

Lemma 3.1. Let $1 \leq n \leq N$. Then we have

$$
c_{n}^{\tau(l)}= \begin{cases}\sigma_{n+1-l}-\sigma_{n-l} & \text { for } 1 \leq l \leq n-1, \\ \sigma_{1} & \text { for } l=n, \\ 0 & \text { for } n+1 \leq l \leq N .\end{cases}
$$

Moreover, in the case $q$ even, we have $c_{n}^{2 l}=0$ for $1 \leq l \leq N$.

Proof. One easily sees that $c_{0}^{j}=0$ for $1 \leq j \leq q-2$ and $c_{0}^{q-1}=\sigma_{1}$. The recurrence relations (i) and (ii) imply that $c_{k}^{1}=0$ for $0 \leq k \leq N-1$ and $c_{N}^{1}=\sigma_{1}$. Using (iii), we obtain $c_{k}^{q-1}=\sigma_{k+1}-\sigma_{k}$ for $1 \leq k \leq N-1$. The value of $c_{n}^{\tau(l)}$ can now be deduced from these results by repeatedly applying (18) in each of the cases $1 \leq l \leq n-1, l=n$ and $n+1 \leq l \leq N$. 
Lemma 3.2. Let $N \geq 2,1 \leq n \leq N$ and $b_{l}$ be defined as in (4). Then we have

$$
\frac{q-6}{q-2} \sigma_{n}+\sum_{j=2}^{q-2} \frac{q-2 j}{q-2} c_{n}^{j}= \begin{cases}\sum_{l=1}^{n-1} b_{l} \sigma_{n-l} & \text { if } n \leq N-1, \\ \frac{6-q}{q-2} \sigma_{1}+\sum_{l=1}^{N-2} b_{l} \sigma_{N-l} & \text { if } n=N .\end{cases}
$$

Proof. First observe that, since $2 \leq \tau(l) \leq q-2$ for $1 \leq l \leq N-1$ and $c_{n}^{\tau(l)}=0$ for $n+1 \leq l \leq N$ by Lemma 3.1

$$
\sum_{j=2}^{q-2}(q-2 j) c_{n}^{j}= \begin{cases}\sum_{l=1}^{n}(q-2 \tau(l)) c_{n}^{\tau(l)} & \text { if } n \leq N-1, \\ \sum_{l=1}^{N-1}(q-2 \tau(l)) c_{n}^{\tau(l)} & \text { if } n=N,\end{cases}
$$

(Note for the case $n=N$ : we have $2 \leq \tau(l) \leq q-2$ only for $1 \leq l \leq N-1$ and $\tau(N)=1$. This makes it necessary to treat this case separately.) The proof follows now straightforwardly with the help of Lemma 3.1 and the equation $(q-2) b_{l}=2(\tau(l)-\tau(l+1))$.

Proof of Theorem 圆, We rewrite equation (17) as follows:

$$
\sigma_{n+1}-\sigma_{n}=\frac{2 q}{q-2}-\frac{2 q}{q-2} \sum_{l=0}^{n} \kappa\left(S_{l}\right)+\sum_{j=2}^{q-2} \frac{q-2 j}{q-2} c_{n}^{j} .
$$

Using $\kappa\left(S_{0}\right)=1-\frac{q-2}{2 q} \sigma_{1}$ and $\frac{2 q}{q-2} \kappa\left(S_{l}\right)=\kappa_{l} \sigma_{l}$ (for the definition of $\kappa_{l}$ see Theorem 21) we obtain

$$
\sigma_{n+1}=\left(\sigma_{1}-\sum_{l=1}^{n} \kappa_{l} \sigma_{l}\right)+\sigma_{n}+\sum_{j=2}^{q-2} \frac{q-2 j}{q-2} c_{n}^{j} .
$$

The recursion formulas in Theorem 2 for $N \geq 2$ and $n \leq N$ follow now directly from (19) and Lemma 3.2 The case $n=N=1$ has to be treated separately: In this case we have $\sum_{j=2}^{q-2}(q-2 j) c_{n}^{j}=0$ and (19) simplifies to $\sigma_{2}=\left(2-\kappa_{1}\right) \sigma_{1}$. The result follows now from the fact that $b_{0}=2$.

It remains to prove the recursion formula (5) for $n>N$. We first consider the case $N \geq 2$. Repeated application of the recurrence relations (i)-(iii) yields

$$
\sum_{j=2}^{q-2} \frac{q-2 j}{q-2} c_{n}^{j}=\left(\sum_{l=0}^{N-1} b_{l} \sigma_{n-l}\right)-\left(\sigma_{n}+\sigma_{n-(N-1)}\right)+\sum_{j=2}^{q-2} \frac{q-2 j}{q-2} c_{n-N}^{j} .
$$

Since $\frac{2 q}{q-2}\left(\kappa\left(B_{n}\right)-\kappa\left(B_{n-N}\right)\right)=\sum_{l=0}^{N-1} \kappa_{n-l} \sigma_{n-l}$, we obtain with (17) and (20)

$$
\begin{aligned}
& \sum_{l=0}^{N-1} \kappa_{n-l} \sigma_{n-l}= \\
= & -\left(\sigma_{n+1}-\sigma_{n}\right)+\left(\sigma_{n-(N-1)}-\sigma_{n-N}\right)+\left(\sum_{l=0}^{N-1} b_{l} \sigma_{n-l}\right)-\left(\sigma_{n}+\sigma_{n-(N-1)}\right),
\end{aligned}
$$

which immediately yields the recursion formula (5) for $n>N \geq 2$. The case $N=1$ is particularly easy and left to the reader. This finishes the proof of Theorem 2 
Now we turn to the proof of Theorem 3. Note first that non-positive vertex curvature implies non-positive corner curvature in the case of face-regular graphs. By [BP2, Theorem 1], both graphs $G, \widetilde{G}$ are therefore without cut-loci and we can apply the recursion formulas in Theorem 2

Lemma 3.3. We have the following estimates for $0 \leq l \leq N-1$ and $k \geq 1$ :

$$
\begin{array}{ll}
b_{l}-\kappa_{k} \geq 2, & \text { if } l=\frac{N-1}{2}(=0) \text { and } q \in\{3,4\}, \\
b_{l}-\kappa_{k} \geq 1, & \text { if } l \neq \frac{N-1}{2} \text { or } q \text { even, } \\
b_{l}-\kappa_{k} \geq 0, & \text { if } l=\frac{N-1}{2}(=1) \text { and } q=5, \\
b_{l}-\kappa_{k} \geq-1, & \text { if } l=\frac{N-1}{2} \text { and } q \geq 7 \text { odd. }
\end{array}
$$

Proof. The case " $l \neq \frac{N-1}{2}$ or $q$ even" follows from $b_{l}=\frac{4}{q-2}$ and $\kappa(v)=1-$ $\frac{q-2}{2 q}|v| \leq 1-3 \frac{q-2}{2 q}$.

Now assume that $l=\frac{N-1}{2}$. Since $b_{l} \geq \frac{4}{q-2}-2$, the previous considerations lead to $b_{l}-\kappa_{k} \geq-1$. If $q=3$ or $q=4$, then $b_{l}=2$ and, consequently, $b_{l}-\kappa_{k} \geq b_{l}=2$. Finally, if $q=5$, then $\kappa(v) \leq 0$ implies that $|v| \geq 4$ and thus $\kappa(v) \leq 1-4 \frac{q-2}{2 q}$. Using this fact leads directly to $b_{l}-\kappa_{k} \geq 0$.

From the above lemma we deduce the following facts:

Lemma 3.4. We have

(a) $b_{0}-\kappa_{k} \geq 1$.

(b) $b_{N-1}-\kappa_{k} \geq 1$ if $N \geq 2$.

(c) $b_{0}-\kappa_{k} \geq 2$ if $q=3$ or $q=4$.

(d) Let $n, N \geq 1,1 \leq k \leq \min \{n, N\}$, and assume that $\gamma_{i} \geq 0$ are mononote non-decreasing for $n-k+1 \leq i \leq n-1$. Then

$$
\sum_{l=1}^{k-1}\left(b_{l}-\kappa_{n-l}\right) \gamma_{n-l} \geq 0 .
$$

Proof. (a), (b) and (c) are trivial consequences of Lemma 3.3. (d) follows immediately from Lemma 3.3, unless we have $k-1 \geq \frac{N-1}{2}$ and $q \geq 7$ odd. But in this case we have $\frac{N-1}{2} \geq 2$ and

$$
\begin{aligned}
\sum_{l=1}^{k-1}\left(b_{l}-\kappa_{n-l}\right) \gamma_{n-l} & \geq\left(b_{1}-\kappa_{n-1}\right) \gamma_{n-1}+\left(b_{\frac{N-1}{2}}-\kappa_{n-\frac{N-1}{2}}\right) \gamma_{n-\frac{N-1}{2}} \\
& \geq \gamma_{n-1}-\gamma_{n-\frac{N-1}{2}} \geq 0,
\end{aligned}
$$

by the monotonicity of $\gamma_{i}$. 
Proof of Theorem [3. The condition $\bar{\kappa}\left(\widetilde{S}_{n}\right) \leq \bar{\kappa}\left(S_{n}\right) \leq 0$ implies that $\widetilde{\kappa}_{n} \leq \kappa_{n}$ for $n \geq 0$, where $\widetilde{\kappa}_{n}=\frac{2 q}{q-2} \bar{\kappa}\left(\widetilde{S}_{n}\right)$. Note that $\sigma_{0}=\widetilde{\sigma}_{0}=1$. Since $\sigma_{1}=\frac{2 q}{q-2}-\kappa_{0}$, $\widetilde{\sigma}_{1}=\frac{2 q}{q-2}-\widetilde{\kappa}_{0}$ we conclude that

$$
\widetilde{\sigma}_{1}-\sigma_{1}=\kappa_{0}-\widetilde{\kappa}_{0} \geq 0=\widetilde{\sigma}_{0}-\sigma_{0} .
$$

The proof is based on induction over $n$ : Assume that $n \geq 1$ and that $\gamma_{k}:=$ $\widetilde{\sigma}_{k}-\sigma_{k}$ is non-negative and monotone non-decreasing for $0 \leq k \leq n$. We aim to show that $\gamma_{n+1} \geq \gamma_{n}$.

We first consider the case $n \leq N$. Then the recursion formula (5) yields

$$
\gamma_{n+1} \geq \gamma_{n}+\sum_{l=1}^{n-1}\left(b_{l}-\kappa_{n-l}\right) \gamma_{n-l},
$$

and $\gamma_{n+1} \geq \gamma_{n}$ follows from Lemma $3.4(\mathrm{~d})$.

Finally, we consider the case $n>N$. If $N \geq 2$, the recursion formula (5), Lemma 3.4 (a,b) and the monotonicity of $\gamma_{k}$ yields

$$
\begin{aligned}
\gamma_{n+1} & \geq \gamma_{n}+\left(\sum_{l=1}^{N-1}\left(b_{l}-\kappa_{n-l}\right) \gamma_{n-l}\right)-\gamma_{n-N} \\
& \geq \gamma_{n}+\sum_{l=1}^{N-2}\left(b_{l}-\kappa_{n-l}\right) \gamma_{n-l} .
\end{aligned}
$$

Again, $\gamma_{n+1} \geq \gamma_{n}$ follows now from Lemma 3.4(d). If $N=1$ (i.e., $q=3$ or $q=4$ ), the recursion formula (5) simplifies considerably and, using Lemma 3.4 (c), we conclude that

$$
\gamma_{n+1} \geq 2 \gamma_{n}-\gamma_{n-1} \geq \gamma_{n}
$$

finishing the proof of Theorem 3

\section{Proof of Theorem 4}

Proof of Theorem 4. We choose a vertex $v_{0} \in V$ and introduce the following functions $m, M: F \rightarrow\{0,1,2, \ldots, \infty\}:$

$$
\begin{aligned}
m(f) & =\min \left\{d\left(w, v_{0}\right) \mid w \in \partial f\right\} \\
M(f) & =\max \left\{d\left(w, v_{0}\right) \mid w \in \partial f\right\} .
\end{aligned}
$$

Note that the face $f$ "opens up" at distance $m(f)$ and "closes up" at distance $M(f)$ from $v_{0}$. We call a face $f$ finite, if $M(f)<\infty$.

The idea of the proof is to "open up" successively every finite face $f \in F$ into an infinigon without violating the vertex bound. In this way, we will build up a comparison tree $T$ with the same vertex bound $p$ and satisfying $\mu(G) \leq \mu(T)$. It turns out, however, that finite faces $f$ with more than one vertex in the sphere $S_{M(f)}\left(v_{0}\right)$ cause problems in this "opening up" procedure. Therefore, we first modify the tessellation $G$ by removing all edges connecting two vertices $v, w$ at the same distance to $v_{0}$. The modified planar graph is denoted by $G_{0}=\left(V_{0}, E_{0}, F_{0}\right)$. The modification $G \rightarrow G_{0}$ is illustrated in Figure 1 (For 

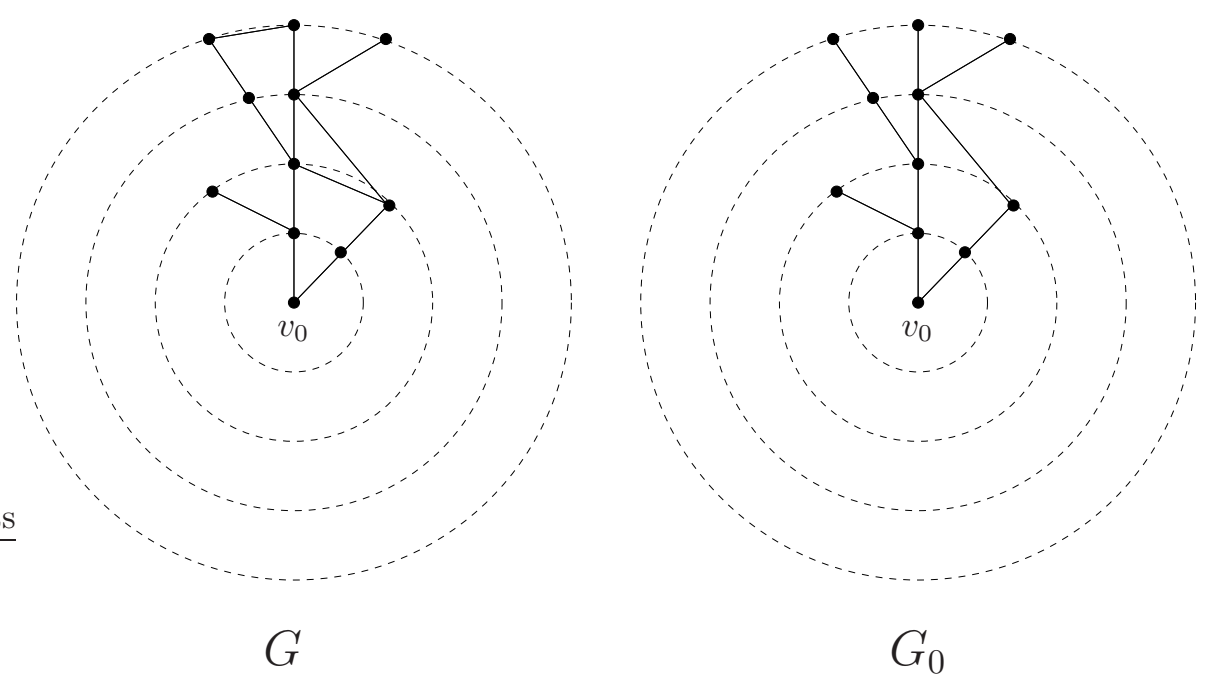

Figure 1: Removing edges between vertices on the same spheres

convenience, the vertices belonging to distance spheres $S_{n}\left(v_{0}\right)$ are arranged to lie on concentric Euclidean circles around $v_{0}$.)

Note that none of the distance relations of the vertices in $G_{0}$ to the vertex $v_{0}$ are changed and that we still have $\operatorname{Cut}\left(v_{0}\right)=\emptyset$. Moreover, the modified graph $G_{0}$ has a new set of faces $F_{0}$. Every finite face $f$ of $G_{0}$ has now even degree, since $f$ opens up at a single vertex in the sphere $S_{m(f)}\left(v_{0}\right)$ and $f$ closes up at a single vertex in the sphere $S_{M(f)}\left(v_{0}\right)$.

We order all finite faces $f_{0}, f_{1}, f_{2}, \ldots$ of $G_{0}$ such that we have

$$
M\left(f_{0}\right) \leq M\left(f_{1}\right) \leq M\left(f_{2}\right) \leq \cdots
$$

Next we explain the first step of our procedure, namely, how to open up $f_{0}$ into an infinigon $\widetilde{f}_{0}$. Let $n=M\left(f_{0}\right) \geq 1$ and $w \in \partial f_{0}$ such that $d\left(w, v_{0}\right)=n$. Since $C\left(v_{0}\right)=\emptyset$, we can find an infinite geodesic ray $w_{0}=w, w_{1}, w_{2}, \cdots \in V$ such that $d\left(w_{i}, v_{0}\right)=n+i$. We may think of $v_{0}$ as being the origin of the plane and of $w_{0}, w_{1}, \ldots$ as being arranged to lie on the positive vertical coordinate axis at heights $n, n+1, \ldots$ with straight edges between them. Now we cut our plane along this geodesic ray, i.e., replace the ray by two parallel copies of the ray and thus preventing the face $f_{0}$ from closing up at distance $n$. In this way, $f_{0}$ becomes an infinigon, which we denote by $f_{0}$. The procedure is illustrated in Figure 2. Note that the vertices $w_{i}$ are replaced by two copies $w_{i}^{(1)}, w_{i}^{(2)}$, such that $w_{i}^{(j)}$ is connected to $w_{i+1}^{(j)}$ for $j=1,2$ and $w_{i}^{(1)}$ inherits all previous neighbors of $w_{i}$ at one side of the ray and $w_{i}^{(2)}$ inherits all previous neighbors of $w_{i}$ at the other side of the ray. In this way we obtain a new planar graph $G_{1}=\left(V_{1}, E_{1}, F_{1}\right)$.

The graph $G_{1}$ is still connected and we obviously have $\mu\left(G_{1}, v_{0}\right) \geq \mu\left(G_{0}, v_{0}\right)=$ $\mu\left(G, v_{0}\right)$. We then carry out the same procedure with the face $f_{1}$ and the face $f_{2}$ and so on. The limit is a connected tree $T$ (since all faces of $T$ are infinigons) satisfying $\mu\left(T, v_{0}\right) \geq \mu\left(G, v_{0}\right)$ with vertex degrees bounded by $p$. Of course, adding branches to make it a $p$-regular tree only further increases the 

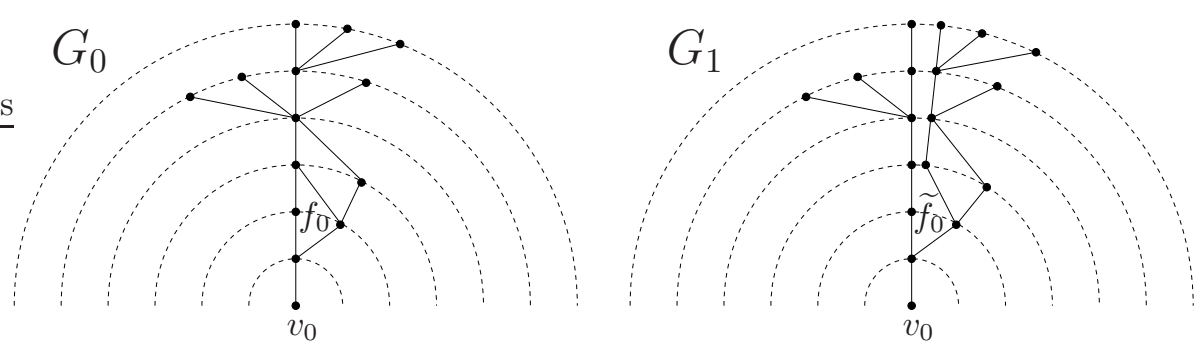

Figure 2: Changing the finite face $f_{0}$ into an infinigon $\widetilde{f}_{0}$

exponential growth. This finishes the proof of Theorem 4.

\section{References}

[BaCS] L. Bartholdi, T. G. Ceccherini-Silberstein, Salem numbers and growth series of some hyperbolic graphs, Geom. Dedicata 90 (2002), 107-114.

[BP1] O. Baues, N. Peyerimhoff, Curvature and geometry of tessellating plane graphs, Discrete Comput. Geom. 25 (2001), no. 1, 141-159.

[BP2] O. Baues, N. Peyerimhoff, Geodesics in Non-Positively Curved Plane Tessellations, Adv. Geom. 6 (2006), no. 2, 243-263.

[CaWa] J. W. Cannon, Ph. Wagreich, Growth functions on surface groups, Math. Ann. 293 (1992), no. 2, 239-257.

[DeVMo] M. DeVos, B. Mohar, An analogue of the Descartes-Euler formula for infinite graphs and Higuchi's conjecture, Trans. Amer. Math. Soc. 359 (2007), no. 7, 32873300 .

[Do] J. Dodziuk, Difference equations, isoperimetric inequality and transience of certain random walks, Trans. Amer. Math. Soc. 284 (1984), no. 2, 787-794.

[DKa] J. Dodziuk, L. Karp, Spectral and function theory for combinatorial Laplacians, Geometry of Random Motion, (R. Durrett, M.A. Pinsky ed.) AMS Contemporary Mathematics, Vol 73, (1988), 25-40.

[DKe] J. Dodziuk, W. S. Kendall, Combinatorial Laplacians and isoperimetric inequality, From Local Times to Global Geometry, Control and Physics, (K. D. Elworthy ed.) Longman Scientific and Technical, (1986), 68-75.

[DL] H. Donnelly, P. Li, Pure point spectrum and negative curvature for noncompact manifolds, Duke Math. J. 46 (1979), no. 3, 497-503.

[FP] W.J. Floyd, S. P. Plotnick, Growth functions on Fuchsian groups and the Euler characteristic, Invent. Math. 88 (1987), no. 1, 1-29.

[Fu1] K. Fujiwara, Growth and the spectrum of the Laplacian of an infinite graph, Tohoku Math. J. (2) 48 (1996), 293-302.

[Fu2] K. Fujiwara, Laplacians on rapidly branching trees, Duke Math J. 83 (1996), no. 1, 191-202.

[GaHuLa] S. Gallot, D. Hulin, J. Lafontaine, Riemannian Geometry, Second edition, Universitext, Springer-Verlag, Berlin, 1990.

[Gro] M. Gromov, Hyperbolic groups, Essays in group theory, 75-263, Math. Sci. Res. Inst. Publ., 8, Springer, New York, 1987.

[HJL] O. Häggström, J. Jonasson, R. Lyons, Explicit isoperimetric constants and phase transitions in the random-cluster model, Ann. Probab. 30 (2002), no. 1, 443-473.

[Hi] Y. Higuchi, Combinatorial curvature for planar graphs, J. Graph Theory 38 (2001), no. 4, 220-229.

[Hi2] Y. Higuchi, Boundary area growth and the spectrum of discrete Laplacian, Ann. Global Anal. Geom. 24 (2003), no. 3, 201-230. 
[HiShi] Y. Higuchi, T. Shirai, Isoperimetric constants of $(d, f)$-regular planar graphs, Interdiscip. Inform. Sci. 9 (2003), no. 2, 221-228.

[HiShi2] Y. Higuchi, T. Shirai, Some spectral and geometric properties for infinite graphs, Discrete geometric analysis, 29-56, Contemp. Math., 347, Amer. Math. Soc., Providence, RI, (2004).

[HLW] S. Hoory, N. Linial, A. Wigderson, Expander graphs and their applications, Bull. Amer. Math. Soc. (N.S.) 43 (2006), no. 4, 439-561.

[Ke] M. Keller, The essential spectrum of the Laplacian on rapidly branching tessellations, Math. Ann. (2009), DOI: 10.1007/s00208-009-0384-y.

[KLPS] S. Klassert, D. Lenz, N. Peyerimhoff, P. Stollmann, Elliptic operators on planar graphs: unique continuation for eigenfunctions and nonpositive curvature, Proc. Amer. Math. Soc. 134, (2005), 1549-1559.

[McK] H. P. McKean, An upper bound to the spectrum of $\Delta$ on a manifold of negative curvature, J. Differential Geometry 4 (1970), 359-366.

[Mo] B. Mohar, Light structures in infinite planar graphs without the strong isoperimetric property, Trans. Amer. Math. Soc. 354 (2002), no. 8, 3059-3074.

[MW] B. Mohar, W. Woess, A survey on spectra of infinite graphs, Bull. London Math. Soc. 21 (1989), no. 3, 209-234.

[St] D. A. Stone, A combinatorial analogue of a theorem of Myers and Correction to my paper: "A combinatorial analogue of a theorem of Myers", Illinois J. Math. 20 (1976), no. 1, 12-21, and Illinois J. Math. 20 (1976), no. 3, 551-554.

[Ura] H. Urakawa, The spectrum of an infinite graph, Canad. J. Math. 52 (2000), no. 5, $1057-1084$.

[We] A. Weber, Analysis of the physical Laplacian and the heat flow on a locally finite graph, http://arxiv.org/abs/0801.0812

[Woe] W. Woess, A note on tilings and strong isoperimetric inequality, Math. Proc. Cambridge Philos. Soc. 124 (1998), no. 3, 385-393.

[Woe2] W. Woess, Random Walks on Infinite Graphs and Groups, Cambridge Tracts in Mathematics, 138, Cambridge University Press, Cambridge, 2000.

[Woj] R. K. Wojciechowski, Stochastic Completeness of Graphs, PhD thesis, 2007, http://arxiv.org/abs/0712.1570

[Woj2] R. K. Wojciechowski, Heat kernel and essential spectrum of infinite graphs, http://arxiv.org/abs/0802.2745 and Indiana Univ. Math. J. (to appear). 